\title{
Percepção socioambiental de moradores que utilizam poços como fonte de abastecimento em um município do arquipélago do Marajó/PA
}

A carência de serviços públicos de gestão e baixos níveis socioeconômicos atrelados à falta de conhecimento e percepção da população quanto aos perigos de se utilizar água contaminada, tem contribuído para maior vulnerabilidade em comunidades rurais, afetando diretamente a qualidade de vida local. Tais fatos motivaram como objetivo da pesquisa verificar o nível de percepção socioambiental de moradores de comunidades rurais do município de Salvaterra, llha de Marajó/PA, que utilizam poços como fonte alternativa de abastecimento hídrico. Realizou-se pesquisa do tipo quali-quantitativa abordando moradores de três comunidades (São Veríssimo, Ceará e Julho). Foi aplicado formulários semiestruturado na escala de Likert de 5 pontos a um total de 136 moradores. 0 perfi socioeconômico do grupo mostrou que predominou o sexo feminino (65\%), na faixa etária de $20-40$ anos, com tempo de escolaridade entre 5 a 10 anos (56\%), vivendo com renda familiar até $\mathrm{R} \$ 500,00$. De um modo geral, a percepção acerca da proteção do recurso e doenças de veiculação hídrica foi considerada alta (7.3), com correlação significativa com escolaridade acima de 5 anos e sexo masculino. Entretanto, apesar de acentuadas percepções, as condições estruturais devido à falta de gestão públicas não favorecem para mudanças na qualidade e comportamento de vida desses moradores.

\section{Socio-environmental perception of residents who use wells as a source of supply in a municipality in the Marajó archipelago/PA}

\begin{abstract}
The lack of public services and management, and low levels of socio-economic challenges in the wake of the lack of knowledge and awareness of the population about the dangers of using contaminated water from wells, and has contributed to greater vulnerability in the rural communities, directly affecting the quality of life of the local. These facts motivate for the purpose of the survey to check the level of the perceived social and environmental to the residents of the rural communities in the municipality of Salvaterra, Island of Marajó island/PA, that use wells as an alternative source of water. Qualitative and quantitative research was carried out addressing residents of three communities (São Verissimo, Ceará e Julho). Semi-structured forms on the 5 point Likert scale were applied to a total of 136 residents. The socioeconomic profile of the group showed that female sex (65\%) predominated, aged $20-40$ years, with schooling time between 5 to 10 years $(56 \%)$, living with a family income of up to $\mathrm{R} \$ 500.00$. In general, the socio-environmental perception of water was considered high, with a significant correlation with schooling over 5 years old and male sex. Meantime, despite sharp perceptions, structural conditions due to a lack of public management do not favor changes in the quality of life these residents.
\end{abstract}

Keywords: Rural environment; Sanitary hygiene; Water; Health; Vulnerable.

Topic: Desenvolvimento, Sustentabilidade e Meio Ambiente

Reviewed anonymously in the process of blind peer.
Received: 05/10/2020

Approved: 26/11/2020
Marcelo Coelho Simões (D)

Universidade do Estado do Pará, Brasil

http://lattes.cnpq.br/5251593133260702

http://orcid.org/0000-0001-6764-1126

marcelo.uepa14@gmail.com

Priscila Sanjuan de Medeiros Sarmento (iD)

Universidade Federal do Pará, Brasil

http://lattes.cnpq.br/5138782013605157

http://orcid.org/0000-0002-5001-9573

priscilasanjuanbio@yahoo.com.br

Marcos Felipe Bentes Cansanção Pereira (ID)

Universidade do Estado do Pará, Brasil

http://lattes.cnpq.br/7697126757065259

http://orcid.org/0000-0002-0306-5454

marcosfelipebentes@gmail.com

6

DOI: 10.6008/CBPC2179-6858.2020.006.0036

\begin{abstract}
Gundisalvo Piratoba Morales
Universidade do Estado do Pará, Brasil

http://lattes.cnpq.br/2923392000120182

http://orcid.org/0000-0002-5930-7053

gundymorales@gmail.com

Cléa Nazaré Carneiro Bichara (it

Universidade do Estado do Pará, Brasil

http://lattes.cnpq.br/2161704040280760

http://orcid.org/0000-0002-2995-0136

cleacarneirobichara@gmail.com
\end{abstract}

Referencing this:

SIMÕES, M. C.; SARMENTO, P. S. M.; PEREIRA, M. F. B. C.; MORALES, G. P.; BICHARA, C. N. C.. Percepção socioambiental de moradores que utilizam poços como fonte de abastecimento em um município do arquipélago do Marajó/PA. Revista Ibero Americana de Ciências Ambientais, v.11, n.6, p.440-450, 2020. DOI:

http://doi.org/10.6008/CBPC2179-6858.2020.006.0036 


\section{INTRODUÇÃO}

A eficiência, a qualidade e a universalidade dos serviços de saneamento básico são fundamentais para o bem viver da população com impactos diretos na saúde pública, no meio ambiente e desenvolvimento econômico (ANTHONJ et al., 2016; ANTHONJ et al., 2017). O aumento dos investimentos nesta área pode ser considerado como parte de uma estratégia de amplo desenvolvimento econômico e social (MADEIRA, 2010).

Para a Organização Pan-americana de Saúde (OPAS), a água e a saúde humana são inseparáveis, uma vez que a baixa qualidade de um recurso hídrico é diretamente relacionada com doenças, seja de maneira direta ou indireta (WHO, 2012). No Brasil, há evidências de que a maioria das pessoas não tem acesso às informações que garantem o uso da água dentro dos parâmetros de segurança o que contribui para o aumento da incidência de doenças de veiculação hídrica nas comunidades rurais do país (SINGER et al., 2012). A qualidade sanitária rural também afeta as populações urbanas podendo provocar alterações nas condições quali-quantitativas dos mananciais o que dificulta o tratamento e fornecimento da água em grandes centros (SHAYO et al., 2015).

Nas regiões menos desenvolvidas, onde as casas não estão conectadas a água encanada, a qualidade das fontes hídricas, como poços e rios, não é formalmente avaliada (ANNAPOORNA et al., 2015). Além disso, o comportamento e saúde não se limitam necessariamente a percepções acerca da qualidade higiênicosanitária, mais envolvem diversas variáveis, como fatores físicos, socioeconômicos, demográficos, além de etnoconhecimentos também fundamentados em crenças religiosas, de saúde e estigmas sociais (RAHMAN et al., 2012). Por exemplo, aspectos de sabor, cor e odor afetam a maneira como os consumidores percebem a qualidade da água e os riscos à saúde associados, que, por sua vez, afetam as escolhas que os consumidores fazem em relação aos serviços e fontes (SHERRY et al., 2019).

Neste contexto, foi realizada uma pesquisa embasada na questão de que, a carência de serviços públicos de gestão e baixos níveis socioeconômicos atrelados à falta de conhecimento e percepção da população quanto aos perigos de se utilizar água contaminada, tem contribuído para maior vulnerabilidade nessas comunidades, afetando diretamente a qualidade de vida local. Tais fatos motivaram como objetivo da pesquisa verificar o nível de percepção socioambiental de moradores de comunidades rurais do município de Salvaterra que utilizam poços como fonte alternativa de abastecimento hídrico.

\section{METODOLOGIA}

\section{Local e população do estudo}

A pesquisa foi realizada em três comunidades rurais do município de Salvaterra, sendo Comunidade São Veríssimo, Comunidade Ceará e Comunidade Julho (Figura 1). 0 município encontra-se na Mesorregião do Marajó, na parte meridional da Ilha de Marajó (LIMA, 2002), de latitude "0045'12" sul e "longitude 48ำ31'00" oeste, a uma distância de 86 km de Belém, capital do Estado do Pará. 


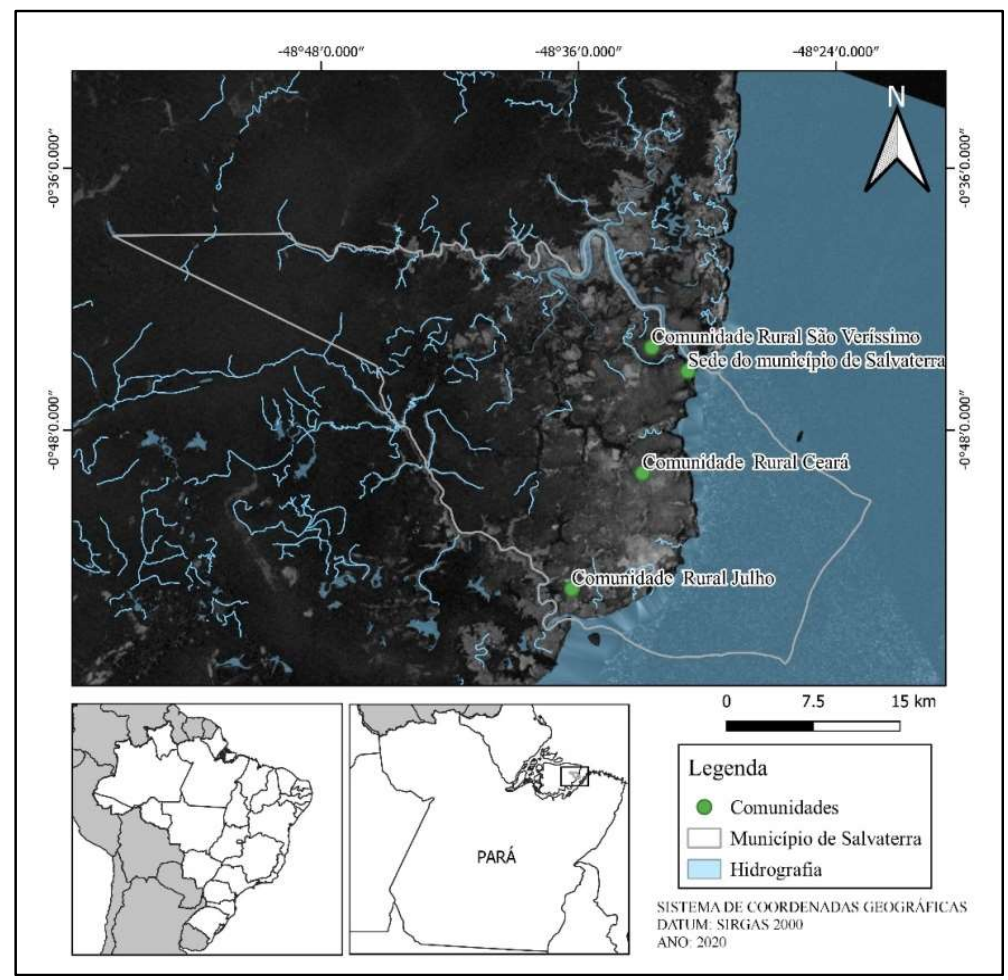

Figura 1: Localização geográfica das três comunidades rurais estudadas no município de Salvaterra- PA.

Segundo o IBGE (2019), a estimativa populacional do município de Salvaterra é de 23.752 pessoas, sendo que destas $37,17 \%$ vivem em áreas rurais. A renda mensal de $51 \%$ das famílias é de cerca de $1 / 2$ salário mínimo. Quanto à educação, a cidade é destaque na região e no estado com uma das mais baixas taxas de analfabetismo (9,04\%), abrangendo escolarização nos anos iniciais em 97,9\% das crianças e adolescentes. Em relação aos serviços básicos, apenas $5 \%$ da população urbana tem esgotamento sanitário, e desta, 57\% possui água encanada. Na área rural, esses serviços não estão presentes. A economia está vinculada a pecuária, particularmente a bubalina, ao extrativismo nas áreas de floresta e à pequena agricultura (IBGE, 2019).

\section{Desenho e coleta de dados}

A análise qualitativa dos dados seguiu a interpretação das informações dos formulários semiestruturados (ALBUQUERQUE et al., 2010; MINAYO et al., 2014). Quanto à abordagem, o estudo foi natureza quali-quantitativa, por meio da análise de conteúdo proposta por Bardin (2011).

As entrevistas e aplicação de formulário semiestruturado ocorreram no mês de maio de 2019 . Utilizando-se o método de coleta aleatória entrevistou-se 136 pessoas, o que representa $32 \%$ da população das três comunidades avaliadas.

O formulário foi composto por questões de identificação social (nome, sexo, naturalidade, escolaridade e religião) e perguntas que versavam sobre a percepção socioambiental dos moradores quanto à água utilizada como fonte de abastecimento doméstico. Os formulários foram elaborados com afirmações semiestruturadas na escala de Likert de 5 pontos, na qual os respondentes indicaram o grau de concordância a afirmativas relacionadas a percepção do objeto (BERMUDES et al., 2016). As afirmações foram organizadas em blocos que abordaram os seguintes temas: bloco 1- Conservação e proteção do Recurso; bloco 2- 
Contaminação; e bloco 3- Doenças por transmissão hídrica. A intensidade de percepção foi classificada em: "baixa", 1-3; "moderada", 4.0-6.9; e "alta", 7-10 (adaptado de BRANDALISE et al., 2009).

\section{Análise dos dados}

Para analisar as diferenças na percepção entre o gênero utilizou-se o teste de hipóteses de MannWhitney. Este teste também foi aplicado para verificar a diferença da percepção entre os moradores que tem acompanhamento de Agentes Comunitários de Saúde ou não. Para comparar a percepção dos impactos entre as comunidades e religião, aplicou-se o teste de Kruskal-Wallis e o pós-teste de Mann-Whitney.

A relação entre a percepção de impacto e as variáveis quantitativas idade; tempo de vida no município (calculado em percentual), renda e idade, foi interpretado através da Análise de Correlação de Kendall. A aplicação dos testes está de acordo com Gotelli et al. (2011) e foram executadas no software estatístico R Development Core Team adotando significância em $p<0,05$.

\section{Aspectos éticos}

Este estudo foi submetido e aprovado pelo Comitê de Ética em Pesquisa da Universidade do Estado do Pará, do Centro de Ciências Biológicas e Saúde (CCBS), com Certificado de Apresentação para Apreciação Ética (CAAE) 11810919.3.0000.8767.

\section{RESULTADOS E DISCUSSÃO}

\section{Perfil socioeconômico dos entrevistados}

Dos 136 moradores entrevistados, nas três comunidades sob estudo, predominou o sexo feminino $65 \%$, com atividades domésticas, cabendo aqueles do sexo masculino atividades voltadas ao sustento da família. Metade destes indivíduos estavam na faixa etária entre 20 a 40 anos de idade (Figura 2).

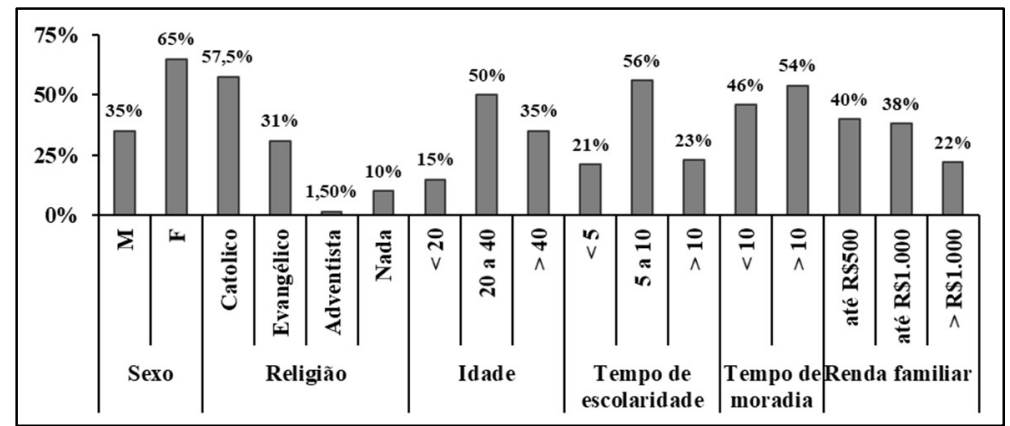

Figura 2: Perfil socioeconômico do público amostral das três comunidades analisadas no estudo.

Quanto ao aspecto religioso, 58\% dos entrevistados afirmaram que são católicos, evangélicos somam $31 \%$ e $11 \%$ não se enquadram nestas duas religiões. Foi importante avaliar tal variável, pois sabe- se que a religião exerce grande influência na construção de princípios e práticas de vivências, o que é respaldado por Aksan et al. (2018) que mostraram que doutrinas religiosas podem influenciar diretamente na interpretação de conhecimentos e saberes de uma determinada população. Do mesmo modo, Herbst et al. (2009) ao realizarem um estudo no Vietnã sobre a mesma temática, observaram que $38 \%$ viviam em prol da religião, 
porém, o uso de água doméstica e comportamento de higiene não são tocados nesses rituais populares.

Nas três comunidades a escolaridade esteve acima de cinco anos, estando entre 5-10 anos na maioria (56\%), essa característica pode se revelar um importante aspecto de proteção às vulnerabilidades correlacionada as condições precárias em áreas rurais na região amazônica. Para Fragkou et al. (2016) o grau de opinião acerca da temática está ligado ao nível de instrução educacional de cada pessoa, onde estes apresentaram uma postura mais flexível em relação aos aspectos socioambientais ligadas ao recurso hídrico, comparados àqueles que afirmaram ter passado menos tempo em ambiente escolar. Embora para Francis et al. (2015) a baixa escolaridade seja um indicativo de problemas sociais, melhores taxas de escolaridades nem sempre são sinônimos de condições adequadas de saneamento, por exemplo.

Foi também observado que 54\% dos moradores locais estão há mais de dez anos com residência fixa nas comunidades, sendo um aspecto relevante, pois novos residentes podem ter antecedentes sobre a qualidade da água em sua localização anterior, o que pode influenciar diretamente no nível de percepção dessa pessoa (AKSAN et al., 2018).

Quanto a variável renda familiar mensal foi observada que a maioria vive com valor menor ou igual à $R \$ 500,00(40 \%)$, seguido dos que vivem com até $R \$ 1.000,00(38 \%)$, respectivamente. Avaliar o nível de percepção e valor de risco econômico tem sido a temática de vários estudos, alguns em contextos diferentes como o de Appiah et al. (2019) em comunidades rurais de Alberta no Canadá, onde prevaleceu aqueles com renda mensal acima de três salários mínimos (51\%) o que não teve influência nas diferenças de percepção ambiental. Mesmo havendo crescimento socioeconômico nos últimos anos em muitos municípios interioranos, localidades rurais ainda vivem em precárias situações sociais, até mesmo pobreza extrema, devido à redistribuição dos recursos ineficaz e falta de oportunidade de trabalho, o que os torna dependentes aos programas sociais (IBGE, 2014).

\section{Níveis de percepção ambiental dos entrevistados}

Observou-se que os usuários confiam nas fontes de água, e que fatores tangíveis, como custo, distância e disponibilidade de recurso hídrico, assim como qualidade, segurança e simplicidade de uso estão presentes no cotidiano de cada um. De acordo com as análises estatísticas, a percepção dos moradores das três comunidades pesquisada é considerada alta (7.3). Tal valor pode ser explicado devido às famílias que já foram afetadas com problemas de saúde, presumivelmente relacionadas à água de baixa qualidade, por vezes essas pessoas conseguem adquirir melhor percepção quanto à utilização deste recurso e até mesmo aos cuidados higiênico-sanitários (BOTZEN et al., 2009). Vásquez et al. (2015) realizando estudos sobre o nível de percepção doméstica e comportamentos quanto à qualidade da água na Nicarágua, onde a principal fonte econômica ainda é a agricultura, observaram que 59\% do universo amostral demonstrou moderado conhecimento quanto às questões ambientais ligadas a água de consumo, e os riscos à saúde, resultados parcialmente semelhantes a estes.

Embora se observe ligeiras diferenças entre as três comunidades estudadas, não houve variação quanto ao nível de percepção entre os moradores. Observou-se diferença na média da renda das famílias 
entre as três comunidades, sendo a maior média, de $\mathrm{R} \$ 1088,78$, encontrada em São Veríssimo, e menor (R\$ 349,73) na comunidade Ceará. A distância dessas comunidades em relação ao centro do município de Salvaterra onde há maior desenvolvimento econômico e ações públicas de gestão, também difere, a comunidade Julho encontra-se há 28 km e São Veríssimo há 8 km do centro.

Com análise das informações obtidas é possível inferir que os direitos sociais adquiridos aliados à escolaridade acima de cinco anos permitiram mostrar boa percepção dos problemas de saúde pública, mesmo na pobreza (Tabela 1), o que pode estar norteando novas atitudes e posturas no comportamento das pessoas (ANTHONJ et al., 2018). Anthonj et al. (2019) avaliando o nível de percepção ambiental sobre água, de moradores de zonas húmidas em uma planície no condado Laikipia, Ewaso e Narok Pântano, no Quênia, observaram resultados parcialmente semelhantes a este, onde $61 \%$ afirmaram estar em áreas expostas as doenças, e puderam relacionar a água como a principal causa de doenças recorrentes na região.

Quanto a variável sexo, o nível de percepção acerca da transmissão de doenças pela água foi significativamente maior entre os homens (Tabela 1).

Tabela 1 Comparação da percepção acerca da proteção do recurso e doenças de veiculação hídrica entre os sexos (S) e locais de moradia (LM).

\begin{tabular}{lllll}
\hline & & Bloco1 & Bloco 2 & Bloco 3 \\
\hline Sexo & Mann-Whitney U & 1766 & 1990 & 1592 \\
& $P$ & 0.13 & 0.64 & $0.02^{*}$ \\
\hline Acompanhamento por & Mann-Whitney U & 1725 & 1864.5 & 1633 \\
agentes Comunitário & $P$ & 0.41 & 0.90 & 0.20 \\
de Saúde & & & & 3.03 \\
\hline Comunidades & Kruskal-Wallis H & 4.74 & 0.30 & 0.21 \\
\hline
\end{tabular}

Bloco 1- conservação e proteção do Recurso; bloco 2- contaminação; bloco 3- doenças por transmissão hídrica.

Supõe que o tempo de escolaridade influenciou para tais resultados, pois a média de estudo dos homens foi de 8,21 anos ante 7,20 anos das mulheres. Mesmo que as mulheres apresentem maior permanência no âmbito familiar, os homens demostraram ter mais informações sobre essas questões, e confirma as muitas mudanças no comportamento e constituição familiar (RAHMAN et al., 2012). Entretanto, segundo Herbst et al. (2009) ao pesquisarem moradores de áreas rurais do Vietnã, observaram que a mulher era a principal responsável pelos cuidados domésticos, estando mais presentes por exemplo no tratamento de doenças de aspecto "comum", e apresentaram maior conhecimento às questões socioambientais vinculadas principalmente ao uso da água.

Quanto a percepção acerca do uso e conservação da água relacionada com a ocorrência de doenças na região, o sexo masculino também apresentou maior grau de percepção (Figura 3).

É importante salientar que, apesar da prevalência do sexo feminino, e grande parte das doenças de quadro "comum" ser tratadas no próprio ambiente familiar, o sexo masculino teve maior percepção. Nos resultados de Anthonj et al. (2018), ao estratificar o público amostral em grupos em áreas rurais no Quênia Central, os pequenos agricultores do sexo masculino demonstraram mais conhecimento, corroborando com estes resultados. 


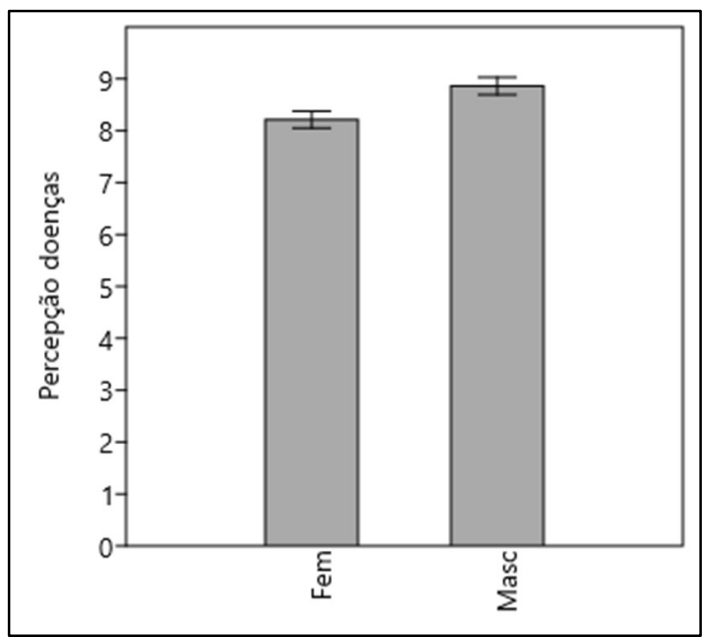

Figura 3: Análise de variância sobre relação da água com doenças entre homens e mulheres.

A percepção acerca da conservação e proteção do recurso está correlacionada negativamente com a idade e a renda, e positivamente com o tempo de escolaridade. Ou seja, quanto maior a idade e a renda, menor o nível de percepção. Quanto maior a escolaridade maior a percepção em relação a conservação do recurso hídrico (Tabela 2). Os resultados corroboram em partes com um estudo realizado por Sah et al. (2017) com famílias rurais do distrito de Saptari-Nepal, onde o tempo de escolaridade esteve associado positivamente aos aspectos higiênico-sanitário ligados à água, e idade e renda não esteve correlacionado com conservação e proteção da água.

Tabela 2: Teste de correlação de Kendall entre a percepção (Bloco 1, 2 e 3) e a idade, escolaridade, tempo de moradia na comunidade e a renda dos entrevistados.

\begin{tabular}{lllll}
\hline & Idade & Escolaridade & Tempo Moradia & Renda \\
\hline Bloco 1 & $-0.11^{*}$ & $0.16^{*}$ & 0.02 & $-0.11^{*}$ \\
Bloco 2 & 0.05 & -0.09 & -0.007 & 0.01 \\
Bloco 3 & $0.12^{*}$ & 0.01 & -0.04 & 0.05 \\
\hline
\end{tabular}

${ }^{*} p<0.05$ = correlações significativas. Bloco 1- conservação e proteção do Recurso; bloco 2- contaminação; bloco 3doenças por transmissão hídrica.

A escola exerce um importante papel no desenvolvimento de uma sociedade, é nela que se desenvolvem valores que fomentam a sociedade, e a falta de investimentos e carência desses espaços acabam por comprometer o conhecimento de uma determinada comunidade (SHERRY et al., 2019). A relação entre a idade e a renda não esteve de acordo com o esperado, uma vez que a escolaridade tende a estar relacionada positivamente com a renda.

Apesar de parte da população conhecer os perigos que acometem a qualidade de vida, as mesmas continuam expostas ao adoecimento e até mesmo agravo de saúde. Netas regiões o acesso ao tratamento de água de qualidade é quase nulo, restando apenas fontes alternativas de abastecimento, por vezes sem nenhum critério higiênico-sanitário (PRÜSS-ÜSTÜN et al., 2014). Segundo o Trata Brasil (2010), é na Amazônia brasileira que se encontram os maiores índices de internações públicas por doenças relacionadas ao saneamento ambiental inadequado devido à má gestão de políticas públicas.

Barcellos et al. (2006) avaliando a qualidade da água e a percepção dos moradores de áreas rurais do município de Lavras- MG, verificou que o nível de percepção ambiental sobre conservação de recurso hídrico é baixo. Os autores ainda atestaram a relação da falta de práticas higiene-sanitárias com a escolaridade. 
$\mathrm{Na}$ área de estudo foi verificado que, a percepção acerca da conservação e proteção do recurso não está correlacionada com o tempo de moradia e renda familiar. Aksan et al. (2018) defendem que, as percepções estão em constantes mudanças, pois novos residentes trazem consigo conceitos diferentes justamente por carregar outros saberes e experiências de sua antiga moradia, e conseguem ter outros olhares sobre o novo lar. Pois, se o agente advém de um local onde a água é conhecida por ser pobre em qualidade, então este inicialmente tende a ter baixa percepção em relação àquele que se mudou recentemente de uma cidade onde a qualidade da água é conhecida por ser boa (UM et al., 2002). Apesar de haver disparidades sociais, não houve diferença daqueles que vivem com um quarto de salário mínimo para os que vivem com dois ou mais. Embora a situação econômica seja diferente, as condições de habitação e sanitárias são semelhantes.

A percepção acerca da contaminação do recurso também é alta e não está relacionada a nenhuma das variáveis quantitativas amostradas. Sherry et al. (2019) avaliando serviços de água de moradores de centros urbanos e rurais na Tanzânia, um país africano conhecido por suas vastas áreas selvagens, observaram que nas áreas rurais independente dos aspectos socioambientais, os participantes estiveram mais atentos as questões ambientais, as mesmas que utilizam fontes alternativas de abastecimento hídrico as caracterizam como insuficientes em quantidade e qualidade. Francis et al. (2015) avaliando a qualidade e percepção da qualidade da água em zonas rurais no sul da Índia, também identificou muitas barreiras à aceitação e uso de intervenções hídricas da população, incluindo a falta de relação percebida entre a água insegura e os impactos negativos à saúde e a resistência a mudanças na estética devido ao tratamento da água, principalmente gosto e odor.

A percepção acerca da transmissão de doenças por veiculação hídrica está correlacionada positivamente com a idade dos entrevistados, pois a percepção é maior naqueles com maior idade. Esses residentes estavam mais atentos às questões ambientais, principalmente voltadas para a saúde, e são eles que mais utilizam meios para tratamento de enfermidades no cotidiano familiar (BONTEMPS et al., 2016).

Segundo Appiah et al. (2019), a maior idade traz consigo mais experiência de vida. Nos achados de Zhen et al. (2019) ao entrevistar moradores para verificar o nível de confiança e risco de consumir água poluída em Xangai, na China, estes apresentaram baixo nível de percepção, além de não haver diferença estatística entre as faixas de idades dos participantes, e concluíram que o maior fator de mudanças no comportamento e percepção de risco é diretamente influenciado pela confiança política local em relação ao suprimento de água doce. Quando existe um alto grau de confiança nas autoridades responsáveis pelo abastecimento de água, a percepção do risco de consumir água é baixa, o que não ocorre na área de estudo, e na Amazônia em geral, onde o recurso geralmente advém de poços ou até mesmo diretamente dos rios (ANA, 2012).

Representações sociais sobre a água de consumo humano são, em geral, elaboradas a partir dos sentidos, sobretudo sabor e visão, conformando um padrão de potabilidade que parece ser sintetizado pela "pureza" da água (STOCKS et al., 2014). O que justifica a sensação de segurança que os moradores geralmente têm sobre esses recursos, mesmo diante da ausência de analises de qualidade e/ou de 
tratamento dos poços dessas áreas rurais.

Nas três comunidades rurais, o público participante percebeu exposição da água como veículo transmissor de doenças, além de boa parte está atento à conservação e proteção do recurso. Diante desse cenário, percebe-se que, de modo geral, o conhecimento da população acerca da relação entre as formas de armazenamento e abastecimento de água com a incidência de determinados tipos de doença não é resumido, mas talvez vivam em situações de vulnerabilidade social por não haver condições de mudar tal realidade, pois, os investimentos em desenvolvimento econômicos e sociais são tímidos, e não contempla a grande maioria dessa população, que está adoecendo pelo simples fato de não ter um sistema público de água, bem como serviços básicos de infraestrutura e saneamento, tornando-as vítimas da improbidade administrativa de gestores públicos.

\section{CONCLUSÕES}

Dentre resultados socioeconômicos, a escolaridade se destacou e esteve intrinsicamente relacionada no nível de percepção, principalmente na conservação e proteção do recurso hídrico que abastecem essas famílias, e confirma a importância de se propagar cada vez mais a educação. No entanto, por mais que se tenha registrado um nível de percepção aceitável nas comunidades de acordo com o método embasado no estudo, as mesmas permanecem em riscos e expostas às doenças veiculadas a água, já que vivem em condições sociais precárias e alta vulnerabilidade, caracterizadas por famílias de baixa renda, carentes de serviços públicos de gestão, e confirma os mais diversos problemas sociais e ambientais presentes na área de estudo.

Apesar de acentuadas percepções, as condições estruturais não favorecem para mudanças na qualidade de vida desses moradores, o que dificulta para que os mesmos passem a ser mediadores e operadores dos bons costumes higiênico-sanitários.

De modo geral, é crucial que se tenha ações de saúde pública local e que estudos com este viés possam ser mais bem difundido e propagado em ambientes de maior vulnerabilidade socioambiental, com o objetivo de apresentar tais resultados aos moradores, representantes comunitários e gestores municipais.

\section{REFERÊNCIAS}

AKSAN, A. M.; VÁSQUEZ, W. F.. Quality Perceptions and Water Treatment Behavior at the Household Level. Water Economics and Policy, California, v.5, n.3, p.33, 2018.

ALBUQUERQUE, U. P.; LUCENA, R. F. P.; CUNHA, L. V. F. C.. Métodos e técnicas na pesquisa etnobiológica $e$ etnoecológica. Recife: NUPPEA, 2010.

ANA. Agência Nacional de Águas. Conjuntura dos recursos hídricos no Brasil: informe especial 2012. Brasília: ANA 2012.

ANNAPOORNA, H.; JANARDHANA, M. R.. Assessment of Groundwater Quality for Drinking Purpose in Rural Areas Surrounding a Defunct Copper Mine. Aquatic Procedia, v.4, n.686, p.685-692, 2015.
ANTHONJ, C.; RECHENBURG, A.; KISTEMANN, T.. Water, sanitation and hygiene in wetlands. A case study from the Ewaso Narok Swamp, Kenya. Int. J. Hyg. Environmental Health, Rockville, v.219, n.10, p.606-616, 2016.

ANTHONJ, C.; RECHENBURG, A.; HÖSER, C.; KISTEMANN, T.. Contracting infectious diseases in Sub-Saharan African wetlands: A question of use? A review. International Journal of Hygiene and Environmental Health, Rockville, v.220, n.7, p.1110-1123, 2017.

ANTHONJ, C.; GITHINJI, S.; KISTEMANN, T.. The impact of water on health and illhealth in a sub-Saharan African wetland: exploring both sides of the coin. Science of the Total Environment, Barcelona, v.624, n.5, p.1411-1420, 
2018.

ANTHONJ, C.; DIEKKRÜGER, B.; BORGEMEISTER, C.; KISTEMANN, T.. Health risk perceptions and local knowledge of water-related infectious disease exposure among Kenyan wetland communities. International Journal of Hygiene and Environmental Health, Rockville, v.222, n.1, p.34-48, 2019.

APPIAH, A.; ADAMOWICZ, W.; LLOYD-SMITH, P.; DUPONT, D.. Reliability of Drinking Water: Risk Perceptions and Economic Value. Water Economics and Policy, Cingapura, v.5, n.2, p.27, 2019.

BARCELLOS, C. M.; ROCHA, M.; RODRIGUES, L. S.; COSTA, C. C.; OLIVEIRA, P. R.; SILVA, I. J.; JESUS, É. F. M.; ROLIM, R. G.. Avaliação da qualidade da água e percepção higiênicosanitária na área rural de Lavras, Minas Gerais, Brasil, 19992000. Caderno Saúde Pública, Rio de Janeiro, v.22, n.9, p.1967-1978, 2006.

BARDIN, L.. Análise de conteúdo. São Paulo: Edições 70, 2011.

BERMUDES, W. L.; SANTANA, B. T.; BRAGA, J. H. O.; SOUZA, P. H.. Tipos de escalas utilizadas em pesquisas e suas aplicações. Revista Vértices, v.18, n.2, p.7-20, 2016.

BONTEMPS, C.; NAUGES, C.. The impact of perceptions in averting-decision models: An application of the special regressormethod to drinking water choices. American Journal of Agricultural Economics, Oxford, v.98, n.1, p.297313,2016

BOTZEN, W. J. W.; AERTS, J. C. J. H.; VAN DEN BERGH, J. C. J. $M$.. Dependence of flood risk perceptions on socioeconomic and objective risk factors. Water Resources Research, v.45, n.10, 2009.

BRANDALISE, B. C. M.; BERTOLINI, G. R. F.; ROJO, C. A.; LEZANA, Á. G. R.; OSMAR, P.. A percepção e o comportamento ambiental dos universitários em relação ao grau de educação ambiental. Gestão \& Produção, São Carlos, v.16, n.2, p.273-285, 2009.

FRAGKOU, M. C.; MCEVOY, J.. Trust matters: Why augmenting water supplies via desalination may not overcome perceptual water scarcity. Desalination, v.397, n.11, p.1-8, 2016

FRANCIS, M.; NAGARAJAN, G.; SARKAR, R.; MOHAN, V.; KANG, G.; BALRAJ, V.. Perception of drinking water safety and factors influencing acceptance and sustainability of a water quality intervention in rural southern India. BMC Public Health, v.15, n.1, p.731, 2015.

GOTELLI, N. J.; ELLISON, A. M.. Princípios de estatística em Ecologia. Porto Alegre: Atmed, 2011.

HERBST, S.; BENEDIKTER, S.; KOESTER, U.; PHAN, N.; BERGER, C.; RECHENBURG, A.; KISTEMANN, T.. Perceptions of water, sanitation and health: a case study from the Mekong Delta, Vietnam. Water Science \& TechnologyWST, v.60, n.3, 2009.

IBGE. Instituto Brasileiro de Geografia e Estatística. Censo Demográfico 2019: características da população e dos domicílios. Rio de Janeiro: IBGE, 2019.
IBGE. Instituto Brasileiro de Geografia e Estatística. PIB municipal 2010. Rio de Janeiro: IBGE, 2014.

LIMA, K.. Variação sazonal da precipitação pluviométrica, temperatura do ar e umidade relativa do ar em ecossistemas de manguezal, floresta e pasto no leste da Amazônia, no período de agosto/2000 a dezembro/2001 Monografia (Bacharelado em Geociências) - Universidade Federal do Pará, Belém, 2002.

MADEIRA, R. F.. O setor de saneamento básico no Brasil e as implicações do marco regulatório para a universalização do acesso. Revista do BNDES, v.33, n.6, p.123-154, 2010.

MINAYO, M. C. S.. O Desafio do Conhecimento: Pesquisa Qualitativa em Saúde. 14 ed. São Paulo: Hucitec-Abrasco, 2014.

PRÜSS-ÜSTÜN, A.; BARTRAM, J.; CLASEN, T.; COLFORD, J. M.; CUMMING, O.; CURTIS, V.; BONJOUR, S.; DANGOUR, A. D.; DE FRANCE, J.; FEWTRELL, L.; FREEMAN, M. C.; GORDON, B.; HUNTER, P. R.; JOHNSTON, R. B.; MATHERS, C.; MÄUSEZAHL, D.; MEDLICOTT, K.; NEIRA, M.; STOCKS, M.; WOLF, J.; CAIRNCROSS, S.. Burden of disease from inadequate water, sanitation and hygiene in low- and middle-income settings: a retrospective analysis of data from 145 countries. Tropica Med. Int. Health, Rockville, v.19, n.8, p.894-905, 2014.

RAHMAN, S. A.; KIELMANN, T.; MCPAKE, B.; NORMAND, C.. Healthcare-seeking behaviour among the tribal people of Bangladesh: can the current health system really meet their needs?. J. Health Popul Nutr., v.30, n.3, p.353-365, 2012.

SAH, R. K.; SAH, P. K.; SAH, J. K.; CHILUWAL, S.; SHAH, S. K. Assessment of the Knowledge, Attitude and Practice Regarding Water, Sanitation and Hygiene among Mothers of Under-five Children in Rural Households of Saptari District, Nepal. American Journal of Public Health Research, v.5, n.5, p.163-169, 2017.

SHAYO, E. H.; RUMISHA, S. F.; MLOZI, M. R. S.; BWANA, V. M.; MAYALA, B. K.; MALIMA, R. C.; MLACHA, T.; MBOERA, L. E. G.. Social determinants of malaria and health care seeking patterns among rice farming and pastoral communities in Kilosa District in central Tanzania. Acta Tropica, v.144, n.01, p.41-49, 2015.

SHERRY, J.; JURAN, L.; KOLIVRAS, K. N.; KROMETIS, L. H.; LING, E. J.. Perceptions of Water Services and Innovations to Improve Water Services in Tanzania. Public Works Management \& Policy, v.24, n.3, p.260-283, 2019.

SINGER, M.; BAER, H.. Introducing Medical Anthropology. A Discipline in Action. 2 ed. Lanham: Rowman \& Littlefield, 2012.

STOCKS, M. E.; OGDEN, S.; HADDAD, D.; ADDISS, D. G.; MCGUIRE, C.; FREEMAN, M. C.. Effect of water, sanitation, and hygiene on the prevention of trachoma: a systematic review and meta-analysis. PLoS Med., Rockville, v.11, n.2, p.1605, 2014.

TRATA BRASIL. Esgotamento sanitário inadequado e impactos na saúde da população. Trata Brasil, 2010.

UM, M.; KWAK, S.; KIM, T.. Estimating willingness to pay for 
improved drinking water quality using averting behavior method with perception measure. Environmental and Resource Economics, v.21, v.3, p.287-302, 2002.

VÁSQUEZ, W. F.; MOZUMDER, P.; FRANCESCHI, D.. Water Quality, Household Perceptions and Averting Behavior: Evidence from Nicaragua. Water Economics and Policy, Cingapura, v.1, n.4, p.21, 2015.
WHO. World Health Organization. WASH POST-2015: Proposed Targets and Indicators for Drinking-water Sanitation and Hygiene. Genebra: WHO, 2014.

ZHEN, N.; BARNETT, J.; WEBBER, M.. Trust and the risk of consuming polluted water in Shanghai, China. Journal of Risk Research, v.22, n.1, p.55-66, 2019.

A CBPC - Companhia Brasileira de Produção Científica (CNPJ: 11.221.422/0001-03) detém os direitos materiais desta publicação. Os direitos referem-se à publicação do trabalho em qualquer parte do mundo, incluindo os direitos às renovações, expansões e disseminações da contribuição, bem como outros direitos subsidiários. Todos os trabalhos publicados eletronicamente poderão posteriormente ser publicados em coletâneas impressas sob coordenação da Sustenere Publishing, da Companhia Brasileira de Produção Científica e seus parceiros autorizados. Os (as) autores (as) preservam os direitos autorais, mas não têm permissão para a publicação da contribuição em outro meio, impresso ou digital, em português ou em tradução. 